\title{
Socioeconomic and Demographic Disparities in Early Surgical Stabilization Following Emergency Department Presentation for Shoulder Instability
}

\author{
Steven L. Bokshan, M.D., Lambert T. Li, B.A., Nicholas J. Lemme, M.D., and \\ Brett D. Owens, M.D.
}

\begin{abstract}
Purpose: To describe which patients are the most likely to undergo surgical management within the same calendar year as their emergency department visit for anterior shoulder instability. Methods: The State Emergency Department Databases and State Ambulatory Surgery and Services Databases from Florida were used. All patients presenting to the emergency department for anterior shoulder subluxation or dislocation between January 1 and September 30, 2017, were selected. Bivariate analysis was performed for associations with demographic variables. A binary logistic regression was performed with all significant factors to assess which were predictors of undergoing surgery the same calendar year. Results: While controlling for all significant factors, we found that patients with recurrent dislocations were 3.14 times more likely to have surgery within the same year $(P=.037)$. Patients younger than 40 years were also 2.04 times more likely to have surgery than those aged 40 years or older $(P<.001)$. White patients were 2.47 times more likely to have surgery than black patients $(P<.001)$. On bivariate analysis, there was an association between greater income quartile and higher odds of undergoing surgery within 30 days. Conclusions: Following an emergency department visit for acute shoulder instability, the following variables were associated with undergoing surgical stabilization within the same calendar year: previous dislocation, age younger than 40 , and white race. Patients living in the greatest income quartile of patients had a significantly greater percentage of patients having surgery within 30 days. This demonstrates that disparities and barriers to care may exist for patients with shoulder instability. Level of Evidence: Level III, Retrospective Comparative Study.
\end{abstract}

A nterior glenohumeral instability is common in the young, active population, with dislocation events occurring as frequently as 1.69 dislocations per 1000 person-years in at-risk populations. ${ }^{1}$ More specifically, younger male patients who participate in contact activities have been shown to have the greatest incidence of recurrent instability, which may ultimately

From the Department of Orthopaedic Surgery, Brown University, Warren Alpert School of Medicine, Providence, Rhode Island, U.S.A.

The authors report the following potential conflicts of interest or sources of funding: B.D.O. reports grants and personal fees from OREF, during the conduct of the study; and personal fees from Mitek, ConMed Linvatec/MTF, outside the submitted work. Full ICMJE author disclosure forms are available for this article online, as supplementary material.

Received April 24, 2020; accepted November 12, 2020.

Address correspondence to Brett D. Owens, M.D., Department of Orthopaedic Surgery, Brown University, Warren Alpert School of Medicine, 1 Kettle Point Ave., Providence, RI 02906.E-mail: owensbrett@gmail.com

(C) 2020 THE AUTHORS. Published by Elsevier Inc. on behalf of the Arthroscopy Association of North America. This is an open access article under the CC BY-NC-ND license (http://creativecommons.org/licenses/by-nc-nd/4.0/).

2666-061X/20580

https://doi.org/10.1016/j.asmr.2020.11.001 necessitate surgical intervention in the form of open or arthroscopic shoulder stabilization. ${ }^{2-12}$

Although much is known about surgical indications and management of anterior shoulder instability, less is known about which patients ultimately present to an orthopaedist for consideration of surgical management. For example, while emergency department (ED) visits for traumatic shoulder dislocation occur at a rate of 23.9 per 100,000 person-years, only a subset of these patients ultimately present to a specialist for discussion of surgical management. ${ }^{3}$ Hutyra et al. ${ }^{4}$ found that among all first-time anterior shoulder dislocations presenting to the ED, only $50 \%$ has been seen in follow-up within 30 days. Moreover, only $29 \%$ of these patients had been presented with high-level medical evidence regarding the management of their shoulder injury over the ensuing 2 -year time period. ${ }^{4}$ Without treatment, these patients may go on to experience recurrent episodes of instability before seeing a specialist.

The purpose of this study was to describe which patients are the most likely to undergo surgical 
management within the same calendar year as their ED visit for anterior shoulder instability. We hypothesized that young male patients with previously documented episodes of instability would be the most likely patients to undergo surgical management within the same calendar year.

\section{Methods}

\section{Data Source}

The 2017 Florida State Ambulatory Surgery and Services Databases (SASD) and State Emergency Department Databases (SEDD) were used in this study. These databases are part of the Healthcare Cost and Utilization Project via the Agency for Healthcare Research and Quality. Discharge abstracts at an encounter level are entered into these databases, along with a unique, anonymized patient identifier that allows tracking of patients across time and health care facilities. Specifically, this database captures more than 300 health care centers in Florida encompassing approximately 2.8 million patients, which represents a large subset of this population. Along with patient demographic information, International Classification of Disease, Tenth Revision (ICD-10) and Current Procedural Terminology codes are included for each patient encounter. The SASD captures outpatient surgeries, and the SEDD captures ED visits.

\section{Patient Selection}

All patients in the SEDD with an ICD-10 code of S43.001 through S43.086 were selected. These diagnosis codes represent all of the codes for shoulder dislocation and subluxation, and they include detail on the side and direction of instability. They also indicate whether it is an initial or subsequent encounter for instability. All patients in the SASD with Current Procedural Terminology codes 23455 (open Bankart repair), 23462 (Latarjet procedure), and 29806 (arthroscopic Bankart repair) were selected. The unique VisitLink patient identifier variable was then used to link patients who presented to the ED and went on to have surgery. Patients who presented to the ED after September 30, 2017, were excluded, as any evaluation and surgery by an orthopaedic surgeon may have occurred in 2018 and thus would not be captured in the 2017 SASD. Cases that were missing demographic variables also were excluded.
Patient age, sex, race, insurance, income quartile based on ZIP code, and instability type were assessed. Instability type included initial dislocation, initial subluxation, and recurrent dislocation. No cases contained ICD-10 codes for recurrent subluxation. The primary outcome variable was surgery within the same calendar year following presentation to the ED and the secondary outcome was the presence of surgery within 30 days of ED presentation.

\section{Statistical Analysis}

An initial bivariate analysis of all demographic variables with surgery following ED presentation was performed. This included analysis of both odds of surgery that year as well as odds of undergoing surgery within 30 days after presentation to the ED. Independent samples $t$-testing and $\chi^{2}$ analysis were performed as appropriate. Variables that were significant under bivariate analysis were included in a binary logistic regression modeling odds of surgery while controlling for all significant factors. $P$ values less than .05 were considered statistically significant. Of note, the $P$ value for instability type was above this threshold under bivariate analysis $(P=.079)$, but it was still included in the binary logistic regression because it was deemed to be approaching significance and of important clinical value. SPSS, version 26.0, was used (IBM Corp., Armonk, NY).

\section{Results}

A total of 7441 patients were identified with the appropriate ICD-10 codes. Of these, 1819 were excluded due to presenting to the ED after September 30,2017 , and 1 patient was excluded due to a missing demographic variable (sex). There was a total of 5621 cases of shoulder instability presenting to the ED from January 1 to September 30, 2017. Of these cases, 3.5\% had a subsequent surgical stabilization that same calendar year. For patients undergoing surgery, 83.6\% had an arthroscopic Bankart repair, $8.7 \%$ had the Latarjet procedure, and $7.7 \%$ had an open Bankart repair. The mean age was 44.6 years old (range, 10-99 years). There was no significant difference in the proportion of patients undergoing each procedure under bivariate analysis with demographic variables.

Patients who underwent surgery were significantly younger than those who were treated nonoperatively (Table 1). The mean age of patients who did not have

Table 1. Comparison of Age for Surgical and Nonsurgical Patients Following a Shoulder Instability Event and Presentation to the Emergency Department

\begin{tabular}{llcccc}
\hline & & & & \multicolumn{2}{c}{$95 \%$ Confidence Interval for Mean } \\
\cline { 5 - 6 } Variable & Group & $\mathrm{N}$ & Mean age & Lower Bound & Upper Bound \\
\hline Age & No surgery & 5426 & 45.0 & 44.5 & 45.6 \\
& Surgery & 195 & 32.3 & 30.1 & 34.4 \\
\hline
\end{tabular}


Table 2. Bivariate Analysis of Categorical Variables for Patients Experiencing a Shoulder Instability Event and Presenting to the Emergency Department

\begin{tabular}{|c|c|c|c|c|}
\hline Group & $\mathrm{N}$ (Total) & No Surgery & Surgery & $P$ Value \\
\hline \multicolumn{5}{|l|}{ Sex } \\
\hline Male & 3582 & $96.0 \%$ & $4.0 \%$ & .005 \\
\hline Female & 2039 & $97.4 \%$ & $2.6 \%$ & \\
\hline \multicolumn{5}{|l|}{ Insurance } \\
\hline Medicare & 1413 & $99.3 \%$ & $0.7 \%$ & $<.001$ \\
\hline Medicaid & 751 & $96.5 \%$ & $3.5 \%$ & \\
\hline Private Insurance & 1794 & $93.1 \%$ & $6.9 \%$ & \\
\hline Other & 1663 & $97.9 \%$ & $2.1 \%$ & \\
\hline \multicolumn{5}{|l|}{ Race } \\
\hline White & 3351 & $96.2 \%$ & $3.8 \%$ & .018 \\
\hline Black & 1058 & $98.0 \%$ & $2.0 \%$ & \\
\hline Hispanic & 1022 & $96.4 \%$ & $3.6 \%$ & \\
\hline Asian & 47 & $91.5 \%$ & $8.5 \%$ & \\
\hline Other & 143 & $95.8 \%$ & $4.2 \%$ & \\
\hline \multicolumn{5}{|l|}{ Income quartile } \\
\hline lst & 2142 & $97.0 \%$ & $3.0 \%$ & .029 \\
\hline 2nd & 1757 & $97.0 \%$ & $3.0 \%$ & \\
\hline $3 r d$ & 1196 & $95.2 \%$ & $4.8 \%$ & \\
\hline 4 th & 526 & $96.1 \%$ & $3.9 \%$ & \\
\hline \multicolumn{5}{|l|}{ Instability type } \\
\hline Initial dislocation & 5274 & $96.5 \%$ & $3.5 \%$ & .079 \\
\hline Initial subluxation & 301 & $98.3 \%$ & $1.7 \%$ & \\
\hline Recurrent dislocation & 46 & $91.3 \%$ & $8.7 \%$ & \\
\hline
\end{tabular}

surgery was 45 versus 32.3 years for patients who were surgically managed $(P<.001)$. There were also differences between groups for the categorical demographic variables (Table 2). Male patients were managed operatively at a rate of $4 \%$ versus $2.6 \%$ for female patients $(P=.005)$. Surgery rates varied significantly by insurance. The rate of surgery among privately insured patients was $6.9 \%$, whereas it was $0.7 \%$ for patients with Medicare $(P<.001)$. Asian patients had the greatest rate of surgery at $8.5 \%$, whereas black patients had the lowest rate at $2.0 \%(P=.018)$. Rates of surgery also varied by income quartile. Patients living in ZIP codes of the lower 2 income quartiles had a 3\% rate of surgery, whereas patients in the highest quartile were managed operatively at a rate of $3.9 \%(P=.029)$. In total, $8.7 \%$ of patients who were diagnosed with a recurrent dislocation in the $\mathrm{ED}$ went on to have surgery, whereas $1.7 \%$ of patients with an initial subluxation were managed operatively $(P=.079)$.

For the surgical group, on analysis of associations between odds of surgery within 30 days and demographic variables, only income quartile of the patient's ZIP code had a significant association (Table 3). There was a stepwise increase in the proportion of patients receiving surgery within 1 month as income quartile increased. Of all patients who had surgery after an instability event, $12.9 \%$ of patients living in the lowest income ZIP codes received surgery within 30 days compared to $40 \%$ of patients living in the highest income ZIP codes $(P=.031)$. A greater proportion of privately insured patients had surgery in this time frame than Medicaid patients, although the difference was only approaching significance $(P=.059)$.

While we controlled for all other significant factors, several of the previously assessed variables held significance in a binary logistic regression (Table 4). Compared with black patients, white patients had 2.47 times greater odds of undergoing surgery the same

Table 3. Bivariate Analysis of Categorical Variables With Odds of Surgery Within 30 Days for Patients Who Had Surgery After Presenting to the Emergency Department With a Shoulder Instability Event

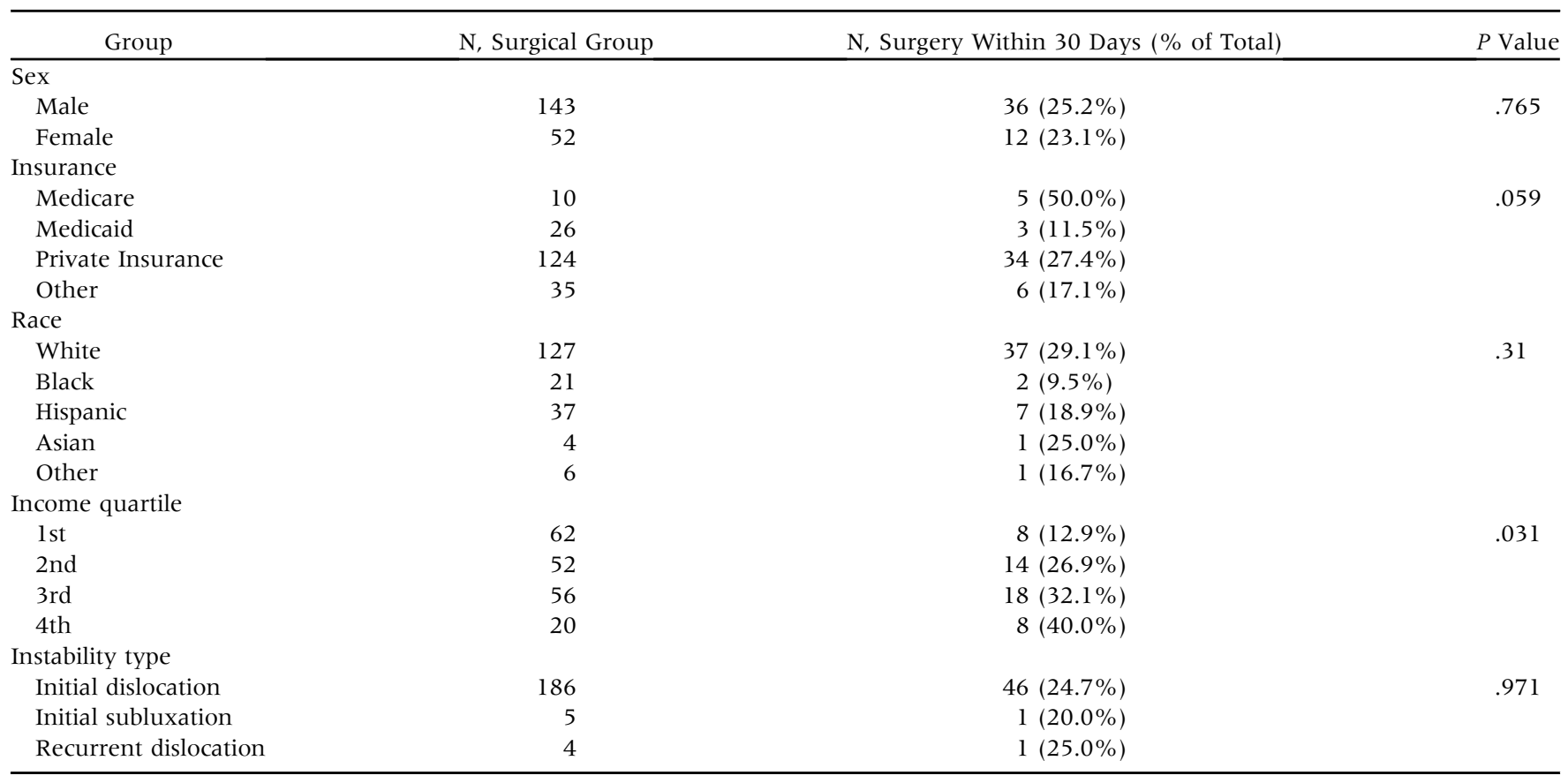


Table 4. Binary Logistic Regression of Odds of Undergoing Surgery After Presenting to the Emergency Department for a Shoulder Instability Event

\begin{tabular}{|c|c|c|c|c|}
\hline \multirow[b]{2}{*}{ Group } & \multirow[b]{2}{*}{ Odds Ratio of Surgery } & \multicolumn{2}{|c|}{ 95\% Confidence Interval } & \multirow[b]{2}{*}{$P$ Value } \\
\hline & & Lower & Upper & \\
\hline Intercept & 0.007 & 0.003 & 0.016 & $<.001$ \\
\hline \multicolumn{5}{|l|}{ Race } \\
\hline Asian & 4.25 & 1.34 & 13.5 & .014 \\
\hline Hispanic & 2.24 & 1.26 & 3.96 & .006 \\
\hline White & 2.47 & 1.49 & 4.09 & $<.001$ \\
\hline Female & 0.865 & 0.610 & 1.23 & .415 \\
\hline Male & Reference & & & \\
\hline \multicolumn{5}{|l|}{ Insurance } \\
\hline Other & 1.91 & 0.888 & 4.11 & .098 \\
\hline Private Insurance & 6.52 & 3.25 & 13.1 & $<.001$ \\
\hline Medicaid & 3.37 & 1.51 & 7.53 & .003 \\
\hline \multicolumn{5}{|l|}{ Age } \\
\hline Younger than 40 & 2.04 & 1.42 & 2.94 & $<.001$ \\
\hline 40 or older & Reference & & & \\
\hline \multicolumn{5}{|c|}{ Income Quartile by ZIP code } \\
\hline 4 th & 0.912 & 0.533 & 1.56 & .738 \\
\hline $3 r d$ & 1.29 & 0.874 & 1.89 & .202 \\
\hline 2nd & 0.843 & 0.574 & 1.24 & .383 \\
\hline lst & Reference & & & \\
\hline
\end{tabular}

NOTE. Significant effects are bolded.

year as ED presentation (95\% confidence interval [CI] 1.49-4.09). Privately insured patients were 6.52 times more likely to have surgery that year than patients with Medicare (95\% CI 3.25-13.1), and Medicaid patients had 3.37 times greater odds of surgery (95\% CI 1.51 7.53) compared with the reference population. Older age was associated with lower odds of surgery. Patients who were younger than 40 years had 2.04 times greater odds of surgery when compared with patients aged 40 years or older (95\% CI 1.42-2.94). Finally, patients who had recurrent dislocations were 3.14 times more likely to undergo surgery than patients who had firsttime instability (95\% CI 1.07-9.23). When controlling for all of these variables, patient sex and income quartile had no significant effects.

\section{Discussion}

Here, we found that previous shoulder dislocation, age younger than 40 years, and white race were associated with undergoing shoulder stabilization within the same calendar year as ED presentation. There is a large body of evidence that demonstrates the effects of a patient's demographic and socioeconomic class not only on health-related outcomes but also on access to specialist care. ${ }^{5}$ Shoulder instability is common, and the management of patients following a first-time instability event is controversial, as some physicians advocate for conservative management following a first-time instability event whereas others recommend early operative intervention, given the high risk of recurrence. ${ }^{6}$ The literature, to date, has focused largely on individual and injury-specific factors, which increase the risk for recurrent instability and early operative management. Conversely, there is a paucity of data investigating which patients ultimately undergo surgical management in a timely manner and whether this is affected by socioeconomic and insurance status. In the present study, we demonstrate that race, insurance status, and socioeconomic class affect which patients undergo surgery for anterior shoulder instability within the same year that they presented to the ED. Specifically, private insurance and recurrent instability were the factors most associated with undergoing surgery that calendar year, followed by age younger than 40 years, male sex, and white or Asian ethnicity. Within the surgical group, there were differences in how quickly patients underwent surgery; patients in the greatest income quartiles were more likely to undergo surgery within 30 days of ED presentation, although it should be noted that surgery within 30 days is not within the standard of care.

Ethnic and racial variations in access and use of health care has been well documented in the field of orthopaedics similar to those found in this study. ${ }^{7-10}$ In 
the present study, African Americans were least likely to undergo surgical stabilization compared with white and Asian patients, who were 2.5 and 4.3 times more likely to undergo surgery, respectively. Similarly, Collins et al. ${ }^{11}$ showed that white patients were more likely to undergo ACL reconstruction compared with nonwhite patients following ACL injury, with an odds ratio (OR) of 1.4. His finding has also been replicated in nonsports surgery, with African American patients being least likely to consider joint arthroplasty despite reporting more severe symptoms and discussing it more frequently with their physicians. ${ }^{10}$ When exploring reasons for lack of surgical management among African American patients, the authors demonstrated the major determinants were related to the subjects' beliefs about the procedural efficacy and the lack of familiarity with this procedure in their community. ${ }^{10}$ In light of this, one potential way to mitigate this disparity as it relates to shoulder instability could include educating the patients on the outcomes following surgical stabilization, particularly during their initial presentation to the emergency room. Indeed, Hutyra et al. ${ }^{4}$ found that among all first-time anterior shoulder dislocations presenting to the ED, only $29 \%$ of these patients had been presented with high-level medical evidence regarding the management of their shoulder injury.

Aside from ethnicity and race, this study also found that patients with private insurance were 6.5 times more likely to undergo surgical stabilization for anterior instability within the same year. This bias has been previously reported in the orthopaedic community, with one survey of orthopaedists finding that $51 \%$ of orthopaedic surgeons considered insurance status to be the primary driver of disparities in access to orthopaedic care. ${ }^{12}$ These beliefs are supported by the fact that less than $50 \%$ of orthopaedic practices in the United States accept Medicaid insurance. ${ }^{13}$ Specifically within the context of shoulder pathology, several studies have found that there was poor access to care among patients with Medicaid. ${ }^{14-17}$ This could also be compounded by the fact that patients with Medicaid have been demonstrated to be twice as likely to fail to arrive for their apointments. ${ }^{18}$ In the present study, we demonstrated patients with private insurance to be more likely to undergo surgery $(6.9 \%$, OR 6.5$)$ during the same calendar year as the instability event compared to Medicaid $(3.5 \%)$ and Medicare patients $(0.7 \%)$. Our findings are consistent with those by Arciero et al., ${ }^{15}$ who demonstrated that patients with nonprivate insurance have longer wait times until surgery, more instability events, and increased bone loss before surgery. Furthermore, when these patients ultimately did undergo surgery, they were more likely to undergo open stabilization than arthroscopic. ${ }^{15}$

Here, we also found that male patients younger than the age of 40 years were more likely to undergo surgical stabilization within the same calendar year. This difference in surgical rates by age and sex is consistent with the risk of instability recurrence following nonsurgical management and is likely related to evidence-based practice by orthopaedic surgeons. ${ }^{2}$ In the present study, we have shown patients younger than the age of 40 years to be twice as likely to undergo surgery compared with those older than 40 years. In addition, male patients were 1.2 times more likely to undergo surgery compared with their female counterparts, likely in an effort to combat recurrence of their instability. These findings are consistent with the risk of instability recurrence. For example, a recent metaanalysis of 1324 patients found an increased risk of recurrent instability following a first-time instability event managed conservatively if they were younger than 40 years of age (OR 13.46) or male sex (OR 2.68). ${ }^{19}$ Many have proposed that this recurrence risk is related to a greater level activity in younger patients, with males being more likely to participate in collision sports. ${ }^{2}$

\section{Limitations}

This study is not without limitations. Inherent to any database study is the possibility that cases of shoulder instability have not been captured due to improper or lack of coding. Likewise, the database nature of this study limits the granularity of knowing which providers assessed for shoulder instability, activity level of these patients, or the proceeding injury events that lead to the emergency visit. In addition, because we were only able to capture patients obtaining follow-up care at Healthcare Cost and Utilization Project outpatient centers, this may under-represent the number of patients that ultimately underwent surgical management for shoulder instability. Furthermore, a larger number patients who initially presented earlier in the year may have had a better chance of going on to surgical intervention that same year. With that being said, we do believe that enough instances of surgical treatment were captured to perform an appropriate regression analysis and control for bias. In addition, as this analysis was limited to Florida for the use of its unique multicenter patient identifiers, it is possible that the practice patterns in this state are not completely representative of every U.S. state. However, given the large number of patients and centers captured in the study, our data are likely reflective of general orthopaedic practice nationwide. Furthermore, it should be noted that patients with Medicare insurance (particularly at a young age) may suffer from an increasing number of comorbidities, thus accounting for lower incidence of surgery. An attempt was made to control for age using multivariate regression. Despite these limitations, we were able to capture a large number of patients to minimize the effects of missing data and, furthermore, the utility of 
this database in examining disparities among various orthopaedic procedures has been well documented. ${ }^{9,20-22}$

\section{Conclusions}

Following an ED visit for acute shoulder instability, the following variables were associated with undergoing surgical stabilization within the same calendar year: previous dislocation, age younger than 40 years, and white race. Patients living in the greatest income quartile of patients had a significantly greater percentage of patients having surgery within 30 days. This demonstrates that disparities and barriers to care may exist for patients with shoulder instability.

\section{References}

1. Owens BD, Dawson L, Burks R, Cameron KL. Incidence of shoulder dislocation in the United States military: Demographic considerations from a high-risk population. J Bone Joint Surg A 2009;91:791-796.

2. Waterman B, Owens BD, Tokish JM. Anterior shoulder instability in the military athlete. Sports Health 2016;8: 514-519.

3. Zacchilli MA, Owens BD. Epidemiology of shoulder dislocations presenting to emergency departments in the United States. Bone Joint Surg A 2010;92:542-549.

4. Hutyra CA, Streufert B, Politzer CS, et al. Assessing the effectiveness of evidence-based medicine in practice: A case study of first-time anterior shoulder dislocations. J Bone Joint Surg A 2019:101.

5. Andrulis DP. Access to care is the centerpiece in the elimination of socioeconomic disparities in health. Ann Intern Med 1998;129:412-416.

6. Lemme NJ, Kuczmarski AS, Goodman AD, Ready LV, Dickens JF, Owens BD. Management and outcomes of inseason anterior shoulder instability in athletes. JBJS Rev 2019;7:e2.

7. Katz JN. Persistence of racial and ethnic differences in utilization and adverse outcomes of total joint replacement. J Bone Joint Surg A 2016;98:1241-1242.

8. Li X, Galvin JW, Li C, Agrawal R, Curry EJ. The impact of socioeconomic status on outcomes in orthopaedic surgery. J Bone Joint Surg A 2020;102:428-444.

9. Schairer WW, Nwachukwu BU, Warren RF, Dines DM, Gulotta LV. Operative fixation for clavicle fractures - socioeconomic differences persist despite overall population increases in utilization. J Orthop Trauma 2017;31:e167-e172.

10. Suarez-Almazor ME, Souchek J, Kelly PA, et al. Ethnic variation in knee replacement: Patient preferences or uninformed disparity? Arch Intern Med 2005;165: $1117-1124$.
11. Collins JE, Katz JN, Donnell-Fink LA, Martin SD, Losina E. Cumulative incidence of ACL reconstruction after ACL injury in adults: Role of age, sex, and race. Am J Sports Med 2013;41:544-549.

12. Adelani MA, O'Connor MI. Perspectives of orthopedic surgeons on racial/ethnic disparities in care. J Racial Ethn Heal Disparities 2017;4:758-762.

13. Merritt Hawkins. 2017 Survey of Physician Appointed Wait Times and Medicare and Medicaid Acceptance Rates; 2017. https://www.merritthawkins.com/newsand-insights/thought-leadership/survey/survey-of-physi cian-appointment-wait-times/\#: :text=The \%202017\% 20Survey \%20 of \% 20Physician, in \% 202004\%2C\% 20prev ious\%20years\%20the. Accessed February 20, 2021.

14. Kirchner GE, Rivers NJ, Balogh EF, et al. Does Medicaid expansion improve access to care for the first-time shoulder dislocator? J Shoulder Elbow Surg 2019;28: 2079-2083.

15. Williams AA, Mancini NS, Kia C, et al. Recurrent shoulder instability: Do morbidity and treatment differ based on insurance? Orthop J Sport Med 2019;7:2325967119841079.

16. Patterson BM, Spang JT, Draeger RW, Olsson EC, Creighton RA, Kamath GV. Access to outpatient care for adult rotator cuff patients with private insurance versus Medicaid in North Carolina. J Shoulder Elbow Surg 2013;22:1623-1627.

17. Rogers MJ, Penvose I, Curry EJ, Galvin JW, Li X. Insurance status affects access to physical therapy following rotator cuff repair surgery: A comparison of privately insured and medicaid patients. Orthop Rev (Pavia) 2019;11: 53-55.

18. Calfee RP, Shah CM, Canham CD, Wong AHW, Gelberman RH, Goldfarb CA. The influence of insurance status on access to and utilization of a tertiary hand surgery referral center. J Bone Joint Surg A 2012;94: 2177-2184.

19. Olds M, Ellis R, Donaldson K, Parmar P, Kersten P. Risk factors which predispose first-time traumatic anterior shoulder dislocations to recurrent instability in adults: A systematic review and meta-analysis. Br J Sports Med 2015;49:913-922.

20. Daniels AH, Arthur M, Esmende SM, Vigneswaran H, Palumbo MA. Incidence and cost of treating axis fractures in the United States from 2000 to 2010. Spine (Phila Pa 1976) 2014;39:1498-1505.

21. Colvin AC, Harrison AK, Flatow EL, Egorova N, Moskowitz A. National trends in rotator cuff repair. J Bone Joint Surg A 2012;94:227-233.

22. Li L, Bokshan SL, Mehta SR, Owens BD. Disparities in cost and access by caseload for arthroscopic rotator cuff Repair: An analysis of 18,616 cases. Orthop J Sport Med 2019;7: 2325967119850503. 